\section{Review: nicotine replacement therapy as assisted "reduction-to-stop" reduces smoking and sustains abstinence in smokers}

\section{OUESTION}

Is nicotine replacement therapy (NRT) effective and safe when used to reduce smoking with the aim of stopping (assisted reduction-to-stop)?

\section{REVIEW SCOPE}

Included studies compared NRT using gum or inhaler with placebo, no treatment, non-NRT drugs, or psychological interventions and reported smoking abstinence in smokers who were unwilling or unable to stop smoking abruptly. Adjunct therapies, where used, had to be included in both treatment groups. Primary outcome was sustained smoking abstinence for 6 months. Other outcomes were sustained smoking reduction or abstinence from 6 weeks to study end and adverse events (AEs).

\section{REVIEW METHODS}

Medline, CINAHL, EMBASE/Excerpta Medica, PsycINFO, Cochrane Library, and Science Citation Index (all 1992-Nov 2007); registries of ongoing trials; and reference lists were searched for published or unpublished randomised controlled trials (RCTs). Experts and drug companies sponsoring NRT trials were contacted. 7 RCTs $(\mathrm{n}=2767$ in analysis, mean age range $42-46$ y, 52\% women, treatment duration 6-18 mo) met the selection criteria: 4 trials used nicotine gum, 2 used inhalers, and 1 allowed choice of gum, inhaler, or patch. 6 trials included behavioural support. The primary outcome, based on individual patient data, included those who stopped smoking for 6 months and those who were abstinent for $<6$ months at study end but would be expected to be abstinent for 6 months if follow-up continued. All trials were high quality (used blinding, intention-to-treat analysis, and accounted for drop-outs).

\section{MAIN RESULTS}

In people unable or unwilling to stop smoking abruptly, metaanalysis showed that NRT-assisted reduction-to-stop smoking resulted in sustained smoking reductions and abstinence (table). Meta-analysis showed that nausea was more frequent in NRT than control groups; groups did not differ for serious AEs (table).

\section{CONCLUSION}

Nicotine replacement therapy is effective for achieving sustained smoking abstinence in smokers who do not intend or are unable to attempt an abrupt quit.

\section{ABSTRACTED FROM}

Moore D, Aveyard P, Connock M, et al. Effectiveness and safety of nicotine replacement therapy assisted reduction to stop smoking: systematic review and meta-analysis. BMJ 2009;338:b1024.

Correspondence to: Dr D Moore, University of Birmingham, Birmingham, UK; d.j.moore@bham.ac.uk

Source of funding: UK Health Technology Assessment Programme (National Institute for Health Research)

Clinical impact ratings: GP/FP/Primary care 6/7; IM/Ambulatory care 6/7; Respirology 6/7; Public health 5/7

NRT-assisted reduction-to-stop smoking $v$ placebo in smokers unable or unwilling to quit abruptly*

\begin{tabular}{|c|c|c|c|c|}
\hline Outcomes & Number of trials $(\mathbf{n})$ & Weighted event rates & RBI $(95 \%$ Cl) & NNT (CI) \\
\hline Smoking abstinence for 6 months & $5(1833)$ & $6.8 \%$ v $3.3 \%$ & $106 \%$ (34 to 215 ) & $29(15$ to 90$)$ \\
\hline Smoking abstinence, week 6 to study end & $7(2767)$ & $1.2 \%$ v $0.4 \%$ & $244 \%$ (48 to 696$)$ & 114 (40 to 579$)$ \\
\hline \multirow[t]{2}{*}{ Sustained smoking reduction, week 6 to study end } & $6(2233)$ & $6.1 \%$ v $1.6 \%$ & $284 \%$ (132 to 535$)$ & 23 (12 to 48$)$ \\
\hline & & & RRI (CI) & NNH (CI) \\
\hline Nausea & $6(2233)$ & $8.6 \%$ v $5.3 \%$ & $63 \%(20$ to 120$)$ & 30 (16 to 96$)$ \\
\hline Serious adverse events & $6(2233)$ & $7.7 \%$ v $7.1 \%$ & $8 \%(-20$ to 45$)$ & NS \\
\hline
\end{tabular}

*NRT, nicotine replacement therapy; NS, not significant; other abbreviations defined in glossary. Weighted event rates, RBI, RRI, NNT, NNH, and Cl calculated from data in article using a fixed-effect model.

T he results of the review by Moore et al show that the "cut-down-to-stop" method increases abstinence from smoking at 6 months compared with placebo (6.8\% v 3.3\%). Relative risks for secondary abstinence and cessation outcomes were similarly encouraging and ranged from 1.3 to 3.8 Adverse events were similar in both groups, although risk of nausea was increased in the NRT group. This may soften our previous warnings about avoiding cigarettes entirely while taking certain forms of NRT. Although one could question the clinical benefit of a therapy that did not work in $93 \%$ of individuals, we are unfortunately resigned to calling this an "effective" result in the challenging world of smoking cessation.
4 studies had data available to assess the efficacy of nicotine replacement gum for the primary outcome. All of these studies were funded by industry: the 2 positive studies were published and the 2 negative studies were not. The weighted mean relative risk for the published studies was 3.7, whereas it was 1.5 and non-significant for the unpublished studies. One wonders if additional unpublished studies, which tend to be more difficult to locate, might exist and substantially alter the results.

Another reason to be wary of gradual reduction of smoking while on NRT is the potential for misinterpretation or overgeneralisation. Might this send the unintended message that it is not actually important to stop smoking completely? Similarly, we should not generalise the results of this review to other forms of NRT, such as nicotine patches or lozenges, since it included few individuals who used these methods.

Although the results of this review are interesting and encouraging, enough questions remain to suggest that we, as practitioners, should proceed with caution.

\section{Brian A Primack, MD, EdM, MS}

University of Pittsburgh

Pittsburgh, Pennsylvania, USA 